\title{
A receding horizon control approach to sampled-data implementation of continuous-time controllers*
}

\author{
Dragan Nešić \\ Department of Electrical and Electronic Engineering \\ The University of Melbourne, Victoria 3010, Australia \\ d.nesic@ee.mu.oz.au \\ Lars Grüne \\ Matematisches Institut, \\ Universität Bayreuth, 95440, Germany \\ lars.gruene@uni-bayreuth.de
}

May 3, 2005

\begin{abstract}
We propose a novel way for sampled-data implementation (with the zero order hold assumption) of continuous-time controllers for general nonlinear systems. We assume that a continuous-time controller has been designed so that the continuous-time closed-loop satisfies all performance requirements. Then, we use this control law indirectly to compute numerically a sampled-data controller. Our approach exploits a model predictive control (MPC) strategy that minimizes the mismatch between the solutions of the sampled-data model and the continuous-time closed-loop model. We propose a control law and present conditions under which stability and sub-optimality of the closed loop can be proved. We only consider the case of unconstrained MPC. We show that the recent results in [6] can be directly used for analysis of stability of our closed-loop system.
\end{abstract}

Keywords: Controller design, stabilisation, sampled-data, nonlinear, receding horizon control, model predictive control.

\section{Introduction}

Nowadays, modern controllers are typically implemented digitally and this fact strongly motivates investigation of sampled-data systems that consist of a continuous-time plant controlled by a discretetime (digital) controller. While tools for analysis and design of linear sampled-data systems are well developed, similar results for nonlinear systems still need development to be as useful as their linear counterparts. A possible approach for sampled-data controller design is to first design a continuoustime controller for the continuous-time plant ignoring sampling and then discretize the obtained controller for digital implementation $[12,14,16]$. The classical discretization methods, such as the Euler, Tustin or matched pole-zero discretization are attractive for their simplicity but they may not perform well in practice since the required sampling rate may exceed the hardware limitations even for linear systems $[1,17]$. This has lead to a range of advanced controller discretization techniques based on optimization ideas that compute "the best discretization" of the continuous-time controller in some sense. A nice account of these optimization based approaches for linear systems has been given in the Bode Lecture by Anderson in [1] and later in the book [12].

We are not aware of a similar optimization based approach for discretization of continuous-time controllers for nonlinear systems. A possible reason for this may be the inherent computational complexity of nonlinear optimal control problems that inevitably require solutions to Hamilton-Jacobi type equations. However, while nonlinear optimal controllers are often impossible to compute in

${ }^{*}$ This research was supported by the Alexander von Humboldt Foundation while the first author was visiting Johan Wolfgang Goete University in Frankfurt am Main and the University of Bayreuth in Bayreuth, Germany as a Humboldt Fellow. The authors would like to thank Mr J. Pannek for completing simulations for the numerical example. 
practice due to the computational burden associated with solving the Hamilton-Jacobi equations, different suboptimal solutions are much more tractable. For example, the receding horizon (or model predictive) control has a manageable computational complexity for relatively large nonlinear problems $[6,10,24]$.

It is the purpose of this paper to introduce a novel receding horizon control scheme that can be used in implementing digitally continuous-time controllers that have been already designed. The cost function that we consider penalizes the difference of solutions of the continuous-time closed-loop system and the sampled-data solutions. In this sense, the control scheme that we consider can be regarded as a nonlinear and sub-optimal version of linear results presented in [1, 12]. For simplicity, we only consider digital implementation of static state feedback controllers and we present results only for unconstrained MPC.

Under appropriate assumptions we prove stability and sub-optimality of our control scheme. We show that stability of our control scheme follows from recent results in [6]. In the same way as in [6] and under appropriate assumptions we can conclude either semi-global and practical asymptotic stability, semi-global asymptotic stability or global exponential stability of the closed loop, where the parameter that we adjust is the optimization horizon and the semi-global property is with respect to the feasibility region for the optimization problem. Then, we show that under appropriate conditions our MPC scheme is inverse optimal (or sub-optimal) in some sense, which is similar to standard MPC results, see [24].

The paper is organized as follows. In Section 2 we present preliminaries and pose the problem we consider. Our control scheme is presented and its stability analysed in Section 3. In Section 4 we consider sub-optimality properties of our MPC scheme. Numerical examples illustrating our approach are presented in Section 5 and conclusions are presented in the final section.

\section{Preliminaries}

The set of real numbers is denoted as $\mathbb{R}$. A function $\gamma: \mathbb{R}_{\geq 0} \rightarrow \mathbb{R}_{\geq 0}$ is called class $\mathcal{G}$ if it is continuous, zero at zero and non-decreasing. It is of class $\mathcal{K}$ if it is continuous, zero at zero and strictly increasing. It is of class $\mathcal{K}_{\infty}$ if it is also unbounded. It is of class $\mathcal{L}$ if it is strictly positive and it is decreasing to zero as its argument tends to infinity. A function $\beta: \mathbb{R}_{\geq 0} \times \mathbb{R}_{\geq 0} \rightarrow \mathbb{R}_{\geq 0}$ is of class $\mathcal{K} \mathcal{L}$ if for every fixed $t \geq 0$ the function $\beta(\cdot, t)$ is of class $\mathcal{K}$ and for each fixed $s>0$ the function $\beta(s, \cdot)$ is class $\mathcal{L}$. Given vectors $\xi, x \in \mathbb{R}^{n}$ we often use the notation $(\xi, x):=\left(\xi^{T} x^{T}\right)^{T}$.

\subsection{Problem formulation}

Consider the plant:

$$
\dot{x}=f(x, u),
$$

where $x \in \mathbb{R}^{n}$ and $u \in \mathbb{U} \subset \mathbb{R}^{m}$ are respectively the state and the control input of the system. Standing assumptions that we will use throughout the paper are as follows:

\section{Standing Assumptions:}

(i) A continuous-time controller $u=u(x)$, such that $u(x) \in \mathbb{U}, \forall x \in \mathbb{R}^{n}$ has been designed for the continuous-time plant (2.1) so that the continuous-time closed-loop system

$$
\dot{x}(t)=f(x(t), u(x(t))) \quad x(0)=x
$$

is (globally) asymptotically stable and satisfies all performance requirements.

(ii) The controller is to be implemented using a sampler and zero order hold. In other words, for a given fixed sampling period $T>0$ the control signal is constant during sampling intervals, i.e. $u(t)=u\left(t_{k}\right)=$ const., $\forall t \in\left[t_{k}, t_{k+1}\right), k \in \mathbb{N}$, where $t_{k}:=k T$.

We will always use $x\left(t, x_{0}\right)$ to denote the solution of the system (2.2) at time $t$ emanating from the initial state $x(0)=x_{0}$. We assume in what follows that $f(x, u(x))$ is locally Lipschitz in $x$ and, hence, for any $x(0)=x_{0}$ the continuous time closed loop system (2.2) has a unique solution. 
Before we pose the problem we consider, we first define what we mean by solutions of the sampleddata system. This definition is the same as the definition of $S$-solution (sampled solution) proposed in [4]. Given an initial state $\xi\left(t_{0}\right)=\xi_{0}$ and a control signal $v(t)=v_{k}, t \in\left[t_{k}, t_{k+1}\right), k \in \mathbb{N}$, the solution of the sampled data system on interval $\left[t_{0}, t_{1}\right]$ is the solution of the continuous time system:

$$
\dot{\xi}(t)=f\left(\xi(t), v_{0}\right), \quad \xi(0)=\xi
$$

Let the solution of this system at time $t_{1}=T$ be denoted as $\xi\left(t_{1}\right)$. Then, solution of the sampled-data system on the time interval $\left[t_{1}, t_{2}\right]$ is the solution of the continuous-time system

$$
\dot{\xi}(t)=f\left(\xi(t), v_{1}\right), \quad \xi\left(t_{1}\right),
$$

and so on. Denote a sequence of controls $v_{k}, k \in[0, M]$ as $v_{[0, M]}$. The solution of the sampled data system at time $t$, starting at $\xi_{0}$ and under the sequence $v_{[0, M]}$ is denoted as $\xi\left(t, \xi_{0}, v_{[0, M]}\right)$ or simply $\xi(t)$ when $\xi_{0}$ and $v_{[0, M]}$ are clear from the context.

The problem that we consider in this paper is as follows:

Find a sampled data controller so that for any given $\xi(0)=\xi$ and $x(0)=x$, the solution $\xi(t)$ of the sampled-data system reproduces the solution $x(t)$ of the continuous-time system "as close as possible".

The optimal solution of the above problem would necessarily involve infinite horizon minimization (optimization) of some measure of the mismatch between sampled-data and continuous-time solutions $(\xi(\cdot)$ and $x(\cdot))$. Since for nonlinear systems infinite horizon optimization typically leads to computationally intractable problems, we will instead investigate suboptimal receding horizon (or model predictive) controllers that are known to be much more manageable computationally. In particular, given a fixed positive integer $M$, the controllers we consider involve minimization $\left(\right.$ in $\left.v_{[0, M-1]}\right)$ of the cost of the form:

$$
J_{M}\left(\xi, x, v_{[0, M-1]}\right):=\sum_{i=0}^{M-1} \int_{t_{i}}^{t_{i+1}} \ell\left(\xi\left(t, \xi, v_{[0, i]}\right)-x(t, x), v_{i}\right) d t+F\left(\xi\left(t_{M}\right), x\left(t_{M}\right)\right)
$$

where $\ell: \mathbb{R}^{n} \times \mathbb{U} \rightarrow \mathbb{R}_{\geq 0}$ and $F: \mathbb{R}^{n} \times \mathbb{R}^{n} \rightarrow \mathbb{R}_{\geq 0}$. In particular, at each sampling interval we solve the following unconstrained optimization problem:

$$
\begin{array}{r}
\hat{u}_{[0, M-1]}=\arg \inf _{v_{[0, M-1]}} J_{M}\left(\xi, x, v_{[0, M-1]}\right) \\
v_{i} \in \mathbb{U} \quad \forall i \in\{0, \ldots, M-1\}
\end{array}
$$

where $(\xi, x)$ are measured at sampling times. Moreover, we implement the controller in a receding horizon fashion where at each sampling interval we apply only the first control

$$
u=u_{M}(\xi, x):=\hat{u}_{0}(\xi, x)
$$

in the optimal sequence $\hat{u}_{[0, M-1]}$. At the next sampling interval the new control sequence is obtained by solving again the optimization problem with new measured states and only the first control in the sequence is actually applied. Note that the receding horizon control law is a static state feedback $u=u_{M}(\xi, x)$ that is implemented in a sampled-data fashion so that the overall closed loop system can be written as follows:

$$
\begin{aligned}
& \dot{\xi}(t)=f\left(\xi(t), u_{M}\left(\xi\left(t_{k}\right), x\left(t_{k}\right)\right)\right) \quad \xi(0)=\xi, \quad t \in\left[t_{k}, t_{k+1}\right) \\
& \dot{x}(t)=f(x(t), u(x(t))) \quad x(0)=x .
\end{aligned}
$$

Several remarks are presented below to further clarify the problem we consider and point out its links with some other approaches in the literature. 
Remark 2.1 One goal of our analysis will be to show that the system (2.7) is asymptotically stable ${ }^{1}$ since then we have

$$
|(\xi(t), x(t))| \leq \beta(|(\xi, x)|, t) \quad \forall t \geq 0,
$$

for some $\beta \in \mathcal{K} \mathcal{L}$, which implies tracking, i.e. $\lim _{t \rightarrow \infty}|\xi(t)-x(t)|=0$. It is obvious from item (i) of our Standing Assumptions that any sampled-data controller that yields $\lim _{t \rightarrow \infty}|\xi(t)|=0$ would also imply tracking even if we do not pose the problem as the minimization of the cost $J_{M}\left(\xi, x, v_{[0, M-1]}\right)$ defined by (2.4). However, model predictive controllers that we propose are sub-optimal in an appropriate sense (see, for instance, Theorem 4.3) and, hence, they are not only achieving tracking but also do so in an appropriate sub-optimal manner.

Remark 2.2 Note that in the system (2.7) the control designer can choose to initialize the bottom continuous-time subsystem in a particular manner, since this is just a reference model to be used in computing the controller $u_{M}(\xi, x)$. For instance, we could measure the initial state of the sampleddata system at the initial time $\xi(0)=\xi$ and then let $x(0)=\xi(0)=\xi$. This makes sense because we would like the sampled-data system to recover as close as possible behavior of the continuous-time system from the same initial condition. Nevertheless, we will present analysis of stability of the system (2.7) that yields bounds on transients for arbitrary initializations, i.e. we will not necessarily assume that $\xi(0)=x(0)$.

Remark 2.3 As we already pointed out, one possible way to tackle this problem is to pose an infinite horizon optimization problem that is aiming at minimizing the difference between the solutions of the closed-loop continuous-time system and the sampled-data system. For linear systems this approach has lead to solutions to different $H_{\infty}$ and $H_{2}$ controller design problems that are tractable [1, 12]. For nonlinear systems, however, this approach necessarily involves Hamilton-Jacobi type equations that are too hard to solve in general and this is the only reason why we do not pursue this approach. Instead, we solve the problem in a receding horizon fashion as described above. This approach is feasible for very large scale nonlinear systems. We are not aware of any references in the literature on Model Predictive Control (MPC) or Receding Horizon Control (RHC) that considered this problem.

Remark 2.4 A brute force (emulation) approach to solving the above problem is to simply implement the following controller:

$$
u(t)=u\left(x\left(t_{k}\right)\right), \quad \forall t \in\left[t_{k}, t_{k+1}\right), \quad k \in \mathbb{N}
$$

and then sample as fast as possible (reduce $T$ ). This approach was shown in [16] to recover the performance of the continuous-time system in an appropriate semi-global practical sense ( $T$ is the parameter that we need to reduce sufficiently). Indeed, emulated controllers are required to have appropriate robustness with respect to the sample and zero order hold implementation in order to preserve stability. This robustness is typically achieved by sufficiently reducing the sampling period. However, due to hardware limitations on the minimum achievable $T$ this approach is often not feasible.

Our controller, on the other hand, will stabilize the system for arbitrarily long sampling periods $T$ if all conditions in our main results hold. Hence, results in this paper are not of the "fast sampling" type that are common in emulation designs for sampled-data systems. Indeed, our results are more similar to the MPC schemes that do not require fast sampling in order to work well ${ }^{2}$.

Remark 2.5 Note that we consider general terminal costs of the form $F\left(\xi\left(t_{M}\right), x\left(t_{M}\right)\right)$ instead of the costs of the form $F\left(\xi\left(t_{M}\right)-x\left(t_{M}\right)\right)$ that may appear to be more natural in this context. However, if we think of the terminal cost as the approximation of the infinite horizon value function, then it is obvious that the form of $F(\cdot, \cdot)$ that we use is more appropriate since the infinite horizon value function

$$
V_{\infty}(\xi, x):=\inf _{v_{[0, \infty)}} \sum_{i=0}^{\infty} \int_{t_{i}}^{t_{i+1}} \ell\left(\xi\left(t, \xi, v_{[0, i]}\right)-x(t, x), v_{i}\right) d t
$$

would not in general have the form $V_{\infty}(\xi-x)$. Nevertheless, our results also apply to the case when $F=F(\xi-x)$.

\footnotetext{
${ }^{1}$ Sometimes we consider semi-global and practical asymptotic stability or global exponential stability.

${ }^{2}$ Of course, the system should not lose stabilizability due to sampling.
} 
Remark 2.6 The problem we consider can be viewed as a special case of a more general problem where we assume that the reference model which we would like our sampled-data closed-loop to track is not necessarily of the form (2.2) but perhaps of the more general form

$$
\dot{x}(t)=g\left(x(t), x\left(t_{k}\right)\right) \quad t \in\left[t_{k}, t_{k+1}\right),
$$

where we assume that this reference model is stable and satisfies all performance requirements.

\subsection{Stability properties of discrete-time MPC schemes}

Our stability results will be heavily based on recently proved stability results of discrete-time MPC schemes in [6]. A unique feature of these results is that the terminal and stage costs do not have be positive definite functions of the state, which is the case for our MPC scheme. Moreover, the terminal cost does not have to be a local control Lyapunov function for the system to show stability ${ }^{3}$. In this section, we summarize results from [6] and in the next section we show how they can be used to analyze stability of the closed loop system (2.7). To this end, we introduce an auxiliary discrete-time problem since [6] deal only with discrete-time systems. Note that given any fixed sampling period $T>0$ the (exact) discrete-time model of the uncontrolled sampled-data system (2.3), (2.2), when it is well defined, has the form:

$$
\begin{array}{rrr}
\xi^{+}=G(\xi, u) & \xi(0)=\xi \\
x^{+}=H(x) & x(0)=x,
\end{array}
$$

where $G(\xi, u):=\xi(T, \xi, u)$ and $H(x):=x(T, x)$. We denote the solutions of the discrete-time model $(2.9)$ as $\xi\left(k, \xi, v_{[0, k-1]}\right)$ and $x(k, x)$ or simply by $\xi_{k}$ and $x_{k}$ when the initial states and the control sequence are clear from the context. Moreover, by introducing the following:

$$
Q(\xi, x, u):=\int_{0}^{T} \ell(\xi(s, \xi, u)-x(s, x), u) d s
$$

we can rewrite the cost (2.4) as follows:

$$
J_{M}(\xi, x, u)=\sum_{i=0}^{M-1} Q\left(\xi_{i}, x_{i}, u_{i}\right)+F\left(\xi_{M}, x_{M}\right)
$$

where $Q$ is defined in (2.10) and $\xi_{i}, x_{i}$ are solutions of the discrete-time system (2.9). Finally, optimization problem (2.5) can be rewritten as follows:

$$
\begin{aligned}
\hat{u}_{[0, M-1]} & =\arg \inf _{v_{[0, M-1]}} \sum_{i=0}^{M-1} Q\left(\xi_{i}, x_{i}, v_{i}\right)+F\left(\xi_{M}, x_{M}\right) \\
v_{i} & \in \mathbb{U} \quad \forall i \in\{0,1, \ldots, M-1\}
\end{aligned}
$$

and the discrete-time model of the closed-loop sampled-data system (2.7) can be written as follows:

$$
\begin{aligned}
\xi^{+} & =G\left(\xi, u_{M}(\xi, x)\right) \\
x^{+} & =H(x)
\end{aligned}
$$

where $u_{M}(\cdot, \cdot)$ comes from $(2.6)$.

Remark 2.7 It is a standard result in the literature to show (under weak assumptions) that stability of the discrete-time model (2.13), (2.14) implies stability of the sampled-data system (2.7) (see, for instance $[26])$.

\footnotetext{
${ }^{3}$ We present a stronger version of results in [6] that is sufficient for our purposes, e.g. we only consider stability of the origin as opposed to stability of arbitrary sets that was considered in [6].
} 
Remark 2.8 Our stability analysis will be based on showing that all conditions of [6] hold for the cost (2.11), and the discrete-time closed-loop (2.13), (2.14) under appropriate conditions on (2.4) and under our standing assumptions. However, in reality the (exact) discrete-time model of the system is not available for controller design and we think of the discrete-time model as an auxiliary (unknown) model via which stability analysis of the system (2.2) can be carried out. Indeed, typically only an approximate discrete-time model can be in general obtained and the controller design based on it. A rigorous framework for controller design based on approximate discrete-time models can be found in $[27,25]$. We do not investigate these implementation issues of our algorithm.

We next adapt appropriate definitions and results from [6] to be applicable for stability analysis of $(2.13),(2.14)$. Some of these definitions can be further relaxed (see [6]) but the version we present suffices for our purposes.

Definition 2.9 Consider the system (2.9) and a function $Q=Q(\xi, x, u)$. The system (2.9) is said to be detectable from $Q$ with respect to $\left(\bar{\alpha}_{W}, \alpha_{W}, \gamma_{W}\right)$ if $\alpha_{W}, \gamma_{W} \in \mathcal{K}_{\infty}$ and $\bar{\alpha}_{W} \in \mathcal{G}$ and there exists a continuous function $W: \mathbb{R}^{2 n} \rightarrow \mathbb{R}_{\geq 0}$ such that for all $(\xi, x) \in \mathbb{R}^{2 n}$ and all $u \in \mathbb{U}$ :

$$
\begin{aligned}
W(\xi, x) & \leq \bar{\alpha}_{W}(|(\xi, x)|) \\
W(G(\xi, u), H(x))-W(\xi, x) & \leq-\alpha_{W}(|(\xi, x)|)+\gamma_{W}(Q(\xi, x, u))
\end{aligned}
$$

Definition 2.10 We will say that the terminal cost $F$ is a control Lyapunov function for the system if it can be decomposed as $F(\xi, x)=F_{N}(\xi, x)=\Gamma(N) \cdot \bar{F}(\xi, x)$, where the function $\Gamma: \mathbb{Z}_{\geq 1} \rightarrow \mathbb{R}_{\geq 1}$ is nondecreasing and unbounded and, moreover, there exist functions $\underline{\alpha}_{F}, \bar{\alpha}_{Q} \in \mathcal{K}_{\infty}$ such that

$$
\underline{\alpha}_{F}(|(\xi, x)|) \leq \bar{F}(\xi, x)
$$

and for every $(\xi, x) \in \mathbb{R}^{2 n}$ there exists $u \in \mathbb{U}$ such that

$$
\begin{aligned}
\bar{F}(G(\xi, u), H(x))-\bar{F}(\xi, x) & \leq 0 \\
Q(\xi, x, u) & \leq \bar{\alpha}_{Q}(|(\xi, x)|) .
\end{aligned}
$$

The following results were proved in [6]:

Theorem 2.11 Suppose that the following conditions hold:

(i) $Q$ and $F$ are continuous;

(ii) $\mathbb{U}$ is bounded;

(iii) The system $(2.9)$ is detectable from $Q(\xi, x, u)$ for some functions $\left(\alpha_{W}, \gamma_{W}, \bar{\alpha}_{W}\right)$;

(iv) The value function is such that for some $\bar{\alpha} \in \mathcal{K}_{\infty}$ we have that $V_{i}(\xi, x) \leq \bar{\alpha}(|(\xi, x)|)$ for all $i \geq 0$ and all $(\xi, x) \in \mathbb{R}^{2 n}$.

Then, for each $M \geq 2$ there exist $\underline{\alpha}_{Y}, \bar{\alpha}_{Y}, \alpha_{Y} \in \mathcal{K}_{\infty}, \beta_{Y} \in \mathcal{K} \mathcal{L}$ and a continuous function $Y_{M}: \mathbb{R}^{2 n} \rightarrow$ $\mathbb{R}_{\geq 0}$ such that for all $(\xi, x) \in \mathbb{R}^{2 n}$ we have:

$$
\begin{aligned}
\underline{\alpha}_{Y}(|(\xi, x)|) & \leq Y_{M}(\xi, x) \leq \bar{\alpha}_{Y}(|(\xi, x)|) \\
Y_{M}\left(G\left(\xi, u_{M}(\xi, x)\right), H(x)\right)-Y_{M}(\xi, x) & \leq-\alpha_{Y}(|(\xi, x)|)+\beta_{Y}(|(\xi, x)|, M) .
\end{aligned}
$$

Moreover, if the following condition also holds:

(v) The terminal cost $F$ is a control Lyapunov function for the system (2.9), 
then there exists a continuous function $Y_{M}$ and $\widehat{\beta}_{Y} \in \mathcal{K} \mathcal{L}$ such that for all $(\xi, x) \in \mathbb{R}^{2 n}(2.17)$ holds and

$$
Y_{M}\left(G\left(\xi, u_{M}(\xi, x)\right), H(x)\right)-Y_{M}(\xi, x) \leq-\alpha_{Y}(|(\xi, x)|)+\widehat{\beta}_{Y}(|(\xi, x)|, \Gamma(M))
$$

Remark 2.12 More details on the conditions used in Theorem 2.11 can be found in [6]. In particular, explicit formulas for computing all bounding functions in Theorem 2.11 are given in [6].

Several direct consequences of Theorem 2.11 are stated next:

Proposition 2.13 Suppose that items (i), (ii), (iii) and (iv) of Theorem 2.11 hold. Then for each pair of strictly positive real numbers $(\Delta, \delta)$ there exists $M_{1}^{*} \in \mathbb{Z}_{\geq 1}$ such that for all $(\xi, x) \in B_{\Delta}$ the solutions of the system (2.13), (2.14) satisfy:

$$
\left|\left(\xi_{k}, x_{k}\right)\right| \leq \max \{\beta(|(\xi, x)|, k), \delta\} \quad \forall k \geq 0 .
$$

Suppose, moreover, that condition (v) of Theorem 2.11 holds. Then, for each pair of strictly positive real numbers $(\Delta, \delta)$ there exists $M_{2}^{*} \in \mathbb{Z}_{\geq 1}$ such that for all $(\xi, x) \in B_{\Delta}$ the solutions of the system (2.13), (2.14) satisfy (2.19).

Remark 2.14 Significance of the condition (v) in the above theorem is that the prediction horizon $M_{2}^{*}$ is different (typically smaller) than $M_{1}^{*}$ for the same given $(\Delta, \delta)$. Condition (v) plays a similar role in all the results that follow.

Proposition 2.15 Suppose that conditions (i), (ii), (iii) and (iv) of Theorem 2.11 hold. Moreover, given $\Delta_{l}>0$ suppose that for $s \in\left[0, \Delta_{l}\right]$ the functions $\alpha_{W}, \bar{\alpha}_{W}, \gamma_{W}, \bar{\alpha}$ from conditions (iii) and (iv) satisfy $\alpha_{W}(s) \geq a_{W} \cdot s^{2}, \bar{\alpha}_{W}(s) \leq \bar{a}_{W} \cdot s^{2}, \gamma_{W}(s) \leq s^{2}, \bar{\alpha}(s) \leq \bar{a} \cdot s^{2}$, where $a_{W}, \bar{a} \in \mathbb{R}_{>0}, \bar{a}_{W} \in \mathbb{R}_{\geq 0}$. Then, there exists $\beta \in \mathcal{K} \mathcal{L}$ such that for each $\Delta>0$, there exists $M_{1}^{*} \in \mathbb{Z}_{\geq 1}$ such that for all $M \geq M_{1}^{*}$ and all $(\xi, x) \in B_{\Delta}$ the solutions of the system (2.13), (2.14) satisfy:

$$
\left|\left(\xi_{k}, x_{k}\right)\right| \leq \beta(|(\xi, x)|, k) \quad \forall k \geq 0 .
$$

Suppose, moreover, that condition (v) of Theorem 2.11 holds with $\underline{\alpha}_{F}(s) \geq \underline{a}_{F} s^{2}, \bar{\alpha}_{Q}(s) \leq \bar{a}_{Q} s^{2}$ for all $s \in\left[0, \Delta_{l}\right]$, where $\underline{a}_{F}, \bar{a}_{Q} \in \mathbb{R}_{>0}$. Then, there exists $\beta \in \mathcal{K} \mathcal{L}$ such that for each $\Delta>0$ there exists $M_{2}^{*} \in \mathbb{Z}_{\geq 1}$ such that for all $(\xi, x) \in B_{\Delta}$ the solutions of the system (2.13), (2.14) satisfy (2.20).

Proposition 2.16 Suppose that all conditions of Corollary 2.15 hold globally and all functions are globally quadratic (i.e. $\Delta_{l}=\infty$ ). Suppose either

- $M>\frac{\bar{a}\left(\bar{a}+\bar{a}_{W}\right)}{a_{W}^{2}}+1$; or

- $F$ is a control Lyapunov function for the system (2.9) with $\underline{\alpha}_{F}(s)=\underline{a}_{F} s^{2}, \bar{\alpha}_{Q}(s)=\bar{a}_{Q} s^{2}$ for $\underline{a}_{F}, \bar{a}_{Q} \in \mathbb{R}_{>0}$ and $M$ is such that $\Gamma(M)>\frac{\bar{a}_{Q} \bar{a}}{\underline{a}_{F} a_{W}}$.

Then, there exist $K \geq 1, \lambda>0$ such that solutions of the system (2.13), (2.14) satisfy:

$$
\left|\left(\xi_{k}, x_{k}\right)\right| \leq K e^{-\lambda k}|(\xi, x)| \quad \forall k \geq 0
$$

Remark 2.17 We stated only the global version of results but with minor changes one can state regional stability results instead. 


\section{Stability}

The purpose of this section is to show that under reasonable assumptions, we can conclude stability of the sampled-data system (2.7). The main results of this section are stated and proved next.

Theorem 3.1 Suppose that the following conditions hold:

(i) $\ell$ and $F$ are continuous;

(ii) $\mathbb{U}$ is bounded;

(iii-a) The continuous-time system (2.2) is globally asymptotically stable;

(iii-b) There exists $r_{0}>0$ and $\gamma \in \mathcal{K}_{\infty}$ with $^{4}$

$$
\ell(y, u) \geq \max \left\{\max _{|x| \leq 2|y|}|f(x, u)|, \gamma(|y|)\right\} \quad \forall|y| \geq r_{0}
$$

(iii-c) $f(\cdot, \cdot)$ and $u(\cdot)$ are locally Lipschitz in their arguments;

(iv) The value function is such that for some $\bar{\alpha} \in \mathcal{K}_{\infty}$ we have that $V_{i}(\xi, x) \leq \bar{\alpha}(|(\xi, x)|)$ for all $i \geq 0$ and all $(\xi, x) \in \mathbb{R}^{2 n}$.

Then, there exists $\beta \in \mathcal{K} \mathcal{L}$ such that for each pair of strictly positive real numbers $(\Delta, \delta)$ there exists $M_{1}^{*} \in \mathbb{Z}_{\geq 1}$ such that for all $(\xi, x) \in B_{\Delta}$ and $M \geq M_{1}^{*}$ the solutions of the system (2.7) satisfy:

$$
|(\xi(t), x(t))| \leq \max \{\beta(|(\xi, x)|, t), \delta\} \quad \forall t \geq 0 .
$$

Suppose, moreover, that:

(v) $F$ is a control Lyapunov function for the system (2.9);

Then, there exists $\beta \in \mathcal{K} \mathcal{L}$ such that for each pair of strictly positive real numbers $(\Delta, \delta)$ there exists $M_{2}^{*} \in \mathbb{Z}_{\geq 1}$ such that for all $(\xi, x) \in B_{\Delta}$ and $M \geq M_{2}^{*}$ the solutions of the system (2.7) satisfy (3.1).

Proof of Theorem 3.1: The proof consists of showing that conditions of Theorem 3.1 imply that all conditions of Proposition 2.13 hold for the underlying discrete-time system. This implies that the discrete-time system (2.13), (2.14) is semiglobally-practically stable in the horizon $M$. Using results from [26] we conclude that the sampled-data system is semiglobally-practically stable in the horizon $M$.

Since we assumed that $\ell$ is continuous, this immediately implies that $Q$ defined in (2.10) is also continuous and item (i) of Proposition 2.13 hold. Moreover, note that items (ii), (iv) and (v) of Theorem 3.1 coincide with corresponding items of Proposition 2.13. Hence, the only thing left to prove is that items (iii-a), (iii-b) and (iii-c) of Theorem 3.1 imply that the item (iii) of Proposition 2.13 holds. The item (iii-a) implies that the discrete-time system (2.14) is globally asymptotically stable. Using the discrete-time Lyapunov converse theorem from (see [18]), we conclude that there exists a Lyapunov function $U(x)$ and $\alpha_{1}, \alpha_{2}, \alpha_{3} \in \mathcal{K}_{\infty}$ such that for all $x \in R^{n}$ we have:

$$
\begin{aligned}
\alpha_{1}(|x|) & \leq U(x) \leq \alpha_{2}(|x|) \\
U(H(x))-U(x) & \leq-\alpha_{3}(|x|) .
\end{aligned}
$$

Note that Proposition 6.1 (see the Appendix) states that conditions (iii-a), (iii-b) and (iii-c) imply that there exists $\alpha_{Q} \in \mathcal{K}_{\infty}$ such that

$$
|x|+Q(\xi, x, u) \geq \alpha_{Q}(|(\xi, x)|) \quad \forall \xi, x, u .
$$

\footnotetext{
${ }^{4} \mathrm{~A}$ similar condition was first used in [11].
} 
Let $\gamma_{W} \in \mathcal{K}_{\infty}$ be arbitrary. Then if we define $W(\xi, x):=U(x)$, we can show that the system (2.9) is detectable from $Q$ with respect to $\left(\alpha_{W}, \gamma_{W}, \bar{\alpha}_{W}\right)$, where

$$
\bar{\alpha}_{W}(s):=\alpha_{2}(s) ; \alpha_{W}(s):=\tilde{\alpha} \circ \frac{1}{2} \alpha_{Q}(s),
$$

and $\tilde{\alpha}(s):=\min \left\{\alpha_{3}(s), \gamma_{W}(s)\right\}$. Indeed, we have:

$$
W(\xi, x)=U(x) \leq \alpha_{2}(|x|) \leq \alpha_{2}(|(\xi, x)|)=: \bar{\alpha}_{W}(|(\xi, x)|) \quad \forall(\xi, x) \in \mathbb{R}^{2 n},
$$

and, hence, (2.15) holds with $\bar{\alpha}_{W}(s):=\alpha_{2}(s)$. Using the definition of $\tilde{\alpha}$, the triangle inequality for class $\mathcal{K}$ functions ${ }^{5}$ and $(3.4)$, we can write:

$$
\begin{aligned}
W(G(\xi, u), H(x))-W(\xi, x) & =U(H(x))-U(x) \\
& \leq-\alpha_{3}(|x|) \\
& =-\alpha_{3}(|x|)-\gamma_{W}(Q(\xi, x, u))+\gamma_{W}(Q(\xi, x, u)) \\
& \leq-\tilde{\alpha}(|x|)-\tilde{\alpha}(Q(\xi, x, u))+\gamma_{W}(Q(\xi, x, u)) \\
& \leq-\tilde{\alpha}\left(\frac{1}{2}(|x|+Q(\xi, x, u))\right)+\gamma_{W}(Q(\xi, x, u)) \\
& \leq-\tilde{\alpha} \circ \frac{1}{2} \alpha_{Q}(|(\xi, x)|)+\gamma_{W}(Q(\xi, x, u))
\end{aligned}
$$

which completes the proof.

Theorem 3.2 Suppose that the following conditions hold:

(i) $\ell$ and $F$ are continuous;

(ii) $\mathbb{U}$ is bounded;

(iii-a) The continuous-time system (2.2) is locally exponentially stable and globally asymptotically stable;

(iii-b) The item (iii-b) of Theorem 3.1 holds and, moreover, there exists $a_{\ell}>0$ and $\Delta_{\ell}>0$ such that

$$
\ell(y, u) \geq a_{\ell}\left(|y|^{2}+|u|^{2}\right) \quad \forall|y| \leq \Delta_{\ell}, u \in \mathbb{U}
$$

(iii-c) $f(\cdot, \cdot)$ and $u(\cdot)$ are locally Lipschitz in their arguments;

(iv) The value function is such that for some locally quadratic $\bar{\alpha} \in \mathcal{K}_{\infty}$ we have that $V_{i}(\xi, x) \leq$ $\bar{\alpha}(|(\xi, x)|)$ for all $i \geq 0$ and all $(\xi, x) \in \mathbb{R}^{2 n}$.

Then, there exists $\beta \in \mathcal{K} \mathcal{L}$ such that for each $\Delta>0$ there exists $M_{1}^{*} \in \mathbb{Z}_{\geq 1}$ such that for all $(\xi, x) \in B_{\Delta}$ and $M \geq M_{1}^{*}$ the solutions of the system (2.7) satisfy:

$$
|(\xi(t), x(t))| \leq \beta(|(\xi, x)|, t) \quad \forall t \geq 0 .
$$

Suppose, moreover, that:

(v) $F$ is a control Lyapunov function for the system (2.9) with locally quadratic $\bar{\alpha}_{Q}$ and $\underline{\alpha}_{F}$;

Then, there exists $\beta \in \mathcal{K} \mathcal{L}$ such that for each $\Delta>0$ there exists $M_{2}^{*} \in \mathbb{Z}_{\geq 1}$ such that for all $(\xi, x) \in B_{\Delta}$ and $M \geq M_{2}^{*}$ the solutions of the system (2.7) satisfy (3.7).

\footnotetext{
${ }^{5} \alpha\left(\frac{1}{2}\left(s_{1}+s_{2}\right)\right) \leq \alpha\left(s_{1}\right)+\alpha\left(s_{2}\right)$ for all $s_{1}, s_{2} \geq 0$.
} 
Proof of Theorem 3.2: This proof follows the same steps as the proof of Theorem 3.1 with the following changes. Since the system (2.2) is locally exponentially stable and globally asymptotically stable (item (iii-a)), one can find a function $U(\cdot)$ so that (3.2) and (3.3) hold with locally quadratic functions $\alpha_{1}, \alpha_{2}, \alpha_{3}$ (we need to prove this for discrete-time systems using the construction that Isidori gives in his second book for continuous-time systems). Moreover, items (iii-b) and (iii-c) imply via Proposition 6.2 that $\alpha_{Q}$ in (3.4) is also locally quadratic. Then, we can pick arbitrary $\gamma_{W}$ that is locally quadratic to conclude that $\bar{\alpha}_{W}, \alpha_{W}$ defined in (3.5) are locally quadratic. Hence, all conditions of Proposition 2.15 hold for the system $(2.13),(2.14)$. The rest of the proof is follows the same steps as the proof of Theorem 3.1 .

Theorem 3.3 Suppose that the following conditions hold:

(i) $\ell$ and $F$ are continuous;

(ii) $\mathbb{U}$ is bounded;

(iii-a) The system (2.2) is globally exponentially stable;

(iii-b) There exists $a_{\ell}>0$ such that

$$
\ell(y, u) \geq a_{\ell}\left(|y|^{2}+|u|^{2}\right) \quad \forall y, u
$$

(iii-c) $f(\cdot, \cdot)$ and $u(\cdot)$ are globally Lipschitz;

(iv) The value function is such that for some $\bar{\alpha}(s):=\bar{a} \cdot s^{2}, \bar{a}>0$ we have that $V_{i}(\xi, x) \leq \bar{\alpha}(|(\xi, x)|)$ for all $i \geq 0$ and all $(\xi, x) \in \mathbb{R}^{2 n}$.

Then, there exist $K \geq 1, \lambda>0$ and $M_{1}^{*} \in \mathbb{Z}_{\geq 1}$ such that for all $(\xi, x) \in \mathbb{R}^{2 n}$ and $M \geq M_{1}^{*}$ the solutions of the system (2.7) satisfy:

$$
|(\xi(t), x(t))| \leq K e^{-\lambda t}|(\xi, x)| \quad \forall t \geq 0 .
$$

Suppose, moreover, that

(v) $F$ is a control Lyapunov function for the system (2.9) with globally quadratic $\bar{\alpha}_{Q}$ and $\underline{\alpha}_{F}$;

Then, there exist $K \geq 1, \lambda>0$ and $M_{2}^{*} \in \mathbb{Z}_{\geq 1}$ such that for all $(\xi, x) \in \mathbb{R}^{2 n}$ and $M \geq M_{2}^{*}$ solutions of the system (2.7) satisfy (3.8).

Proof of Theorem 3.3: This proof follows the same steps as the proof of Theorem 3.2 with the following changes. Since the system (2.2) is globally exponentially stable (item (iii-a)), one can find a function $U(\cdot)$ so that (3.2) and (3.3) hold with globally quadratic functions $\alpha_{1}, \alpha_{2}, \alpha_{3}$ (cite some standard text). Moreover, items (iii-b) and (iii-c) imply via Proposition 6.2 that $\alpha_{Q}$ in (3.4) is also globally quadratic. Then, we can pick an arbitrary globally quadratic $\gamma_{W}$ to conclude that $\bar{\alpha}_{W}, \alpha_{W}$ defined in (3.5) are also globally quadratic. Hence, all conditions of Proposition 2.16 hold for the system $(2.13),(2.14)$. The rest of the proof follows the same steps as the proof of Theorem 3.1.

Remark 3.4 Note that although we are considering an unconstrained optimization problem, the conditions in Theorem 3.3 may not be satisfied for all $(\xi, x) \in \mathbb{R}^{2 n}$ and $u \in \mathbb{U}$.

First, controllability of the sampled-data (and, hence, discrete-time) system may be lost due to sampling and as a result some of our conditions will be impossible to satisfy. For example, it was shown in [6] that asymptotic controllability of the system would be sufficient for the condition (iv) in Theorem 3.3 to hold. Hence, if the discrete-time system is not asymptotically controllable ${ }^{6}$ due to sampling then the condition (iv) may not be possible to satisfy. Moreover, due to possible finite escape times, the solutions of the sampled-data system may not be defined for all initial states and inputs. Hence, our conditions (if satisfied) implicitly guarantee that the system is asymptotically controllable and the solutions of the sampled-data system exist for the given sampling period.

\footnotetext{
${ }^{6}$ For appropriate definitions of asymptotic controllability see [6].
} 
It is standard to show that both of these issues disappear if we sample fast enough (this is not necessary though!). In particular, one can show that if the continuous-time system is asymptotically controllable then given arbitrarily large compact set $N$ there exists $T^{*}>0$ such that for all sampling periods $T \in\left(0, T^{*}\right)$ the solutions exist for all $(\xi, x) \in N u \in \mathbb{U}$ and the system is controllable on the set. Hence, given an arbitrary compact set $N$, we can first adjust the sampling period $T$ to ensure existence of solutions and asymptotic controllability on $N$ and then adjust the horizon length to ensure that $N$ is a subset of the domain of attraction of (2.7).

For simplicity, we will not consider these issues and we will assume that the sampled-data and discrete-time models are well defined everywhere and controllable.

Remark 3.5 We note that [6] provides explicit bounds for the horizon length to guarantee appropriate stability properties of the underlying discrete-time system (see, for instance, Proposition 2.16). We did not present such bounds because some of our proofs are not constructive. In particular, given conditions (iii-a), (iii-b) and (iii-c), we do not provide in general an explicit construction of $\alpha_{W}$ that we use to show that the system (2.9) is detectable from $Q$.

Remark 3.6 From the proof of Theorem 3.1 we can see that we can pick arbitrary $\gamma_{W}$ when proving the detectability property of the system. In particular, we can always choose $\gamma_{W}(s) \leq s, \forall s \geq 0$. If we make this choice, then results of [6] state that the Lyapunov function for the closed-loop discrete time system $(2.13),(2.14)$ can be chosen in the following manner:

$$
V(\xi, x):=V_{M}(\xi, x)+U(x),
$$

where $U$ comes from $(3.2),(3.3)$.

Remark 3.7 Note that our problem can be interpreted as a tracking problem where we want trajectories of the sampled-data system to track the trajectories of the continuous-time model. This set up is standard in the output tracking literature where the continuous-time model is termed an exogenous model (e.g. see [9]). We note, however, that it is typically assumed in the output tracking literature that the exogenous system is Poisson stable, which does not hold in our case. Consequently, our controller, which is a static state feedback controller, does not have the well known internal model structure that is known to be necessary for tracking when the exogenous system is Poisson stable.

Example 3.8 (This example was taken from [15].) We illustrate the performance of our algorithm by a third order Galerkin approximation of the Moore-Greitzer model

$$
\begin{aligned}
\dot{\phi} & =-\psi-\frac{3}{2} \phi^{2}-\frac{1}{2} \phi^{3}-3 R \phi-3 R \\
\dot{\psi} & =-u \\
\dot{R} & =-\sigma R\left(R+2 \phi+\phi^{2}\right)
\end{aligned}
$$

A stabilizing continuous time feedback has been obtained in [20] and is given by

$$
\begin{aligned}
u= & -\left(c_{1}-3 \phi\right)\left(-\psi-\frac{3}{2} \phi^{2}-\frac{1}{2} \phi^{3}-3 \phi R-3 R\right)+c_{2}\left(\psi-c_{1} \phi+\frac{3}{2} \phi^{2}+3 R\right)-\phi \\
& -3 \sigma R\left(R+2 \phi+\phi^{2}\right)
\end{aligned}
$$

Using the parameters $\left(c_{1}, c_{2}, \sigma\right)=(1,2,2)$, initial value $(\phi, \psi, R)=(6,25,1)$ and sampling rate $T=$ 0.05 , Figure 3.1 shows that using emulation of $u$ according to Remark 2.4 stabilizes the system. One can also see that the computed control sequence using the model predictive control algorithm improves on the emulation design since the trajectories of the system stay close to solutions of the system with continuous feedback. In this case the length of the horizon was chosen to be $10 T$ and the solution can be improved by enlarging the horizon. Note that we achieved the results from Figure 3.1 without using a Lyapunov function for the terminal cost.

Using parameters $\left(c_{1}, c_{2}, \sigma\right)=(1,50,2)$ and again $T=0.05$, for the same initial value it can be seen from Figure 3.2 that emulation of $u$ does not stabilize the system. However, also in this case the implemented MPC scheme is able to generate a control sequence that stabilizes the system and keeps the sampled-data solution close to the continuous time one. 

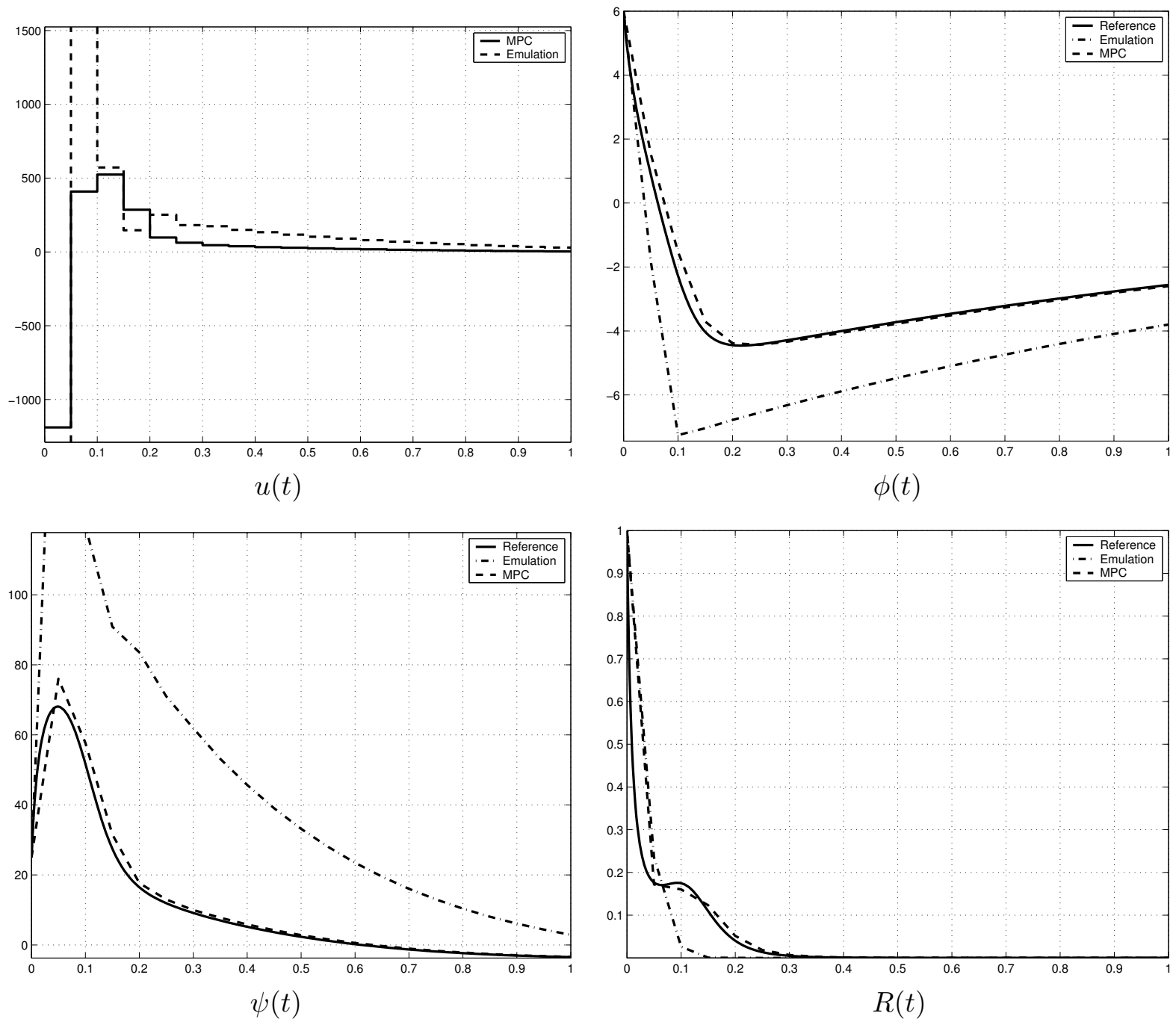

Figure 3.1: Improvement of MPC on stable emulated controller

\section{Optimality}

It is also interesting to note that under extra assumptions, our control law is inverse optimal in some sense. In other words, there exists another infinite horizon cost with respect to which our controller is optimal. This infinite horizon cost involves the difference between trajectories of the continuous time system and the sampled-data system. This is similar to standard results in model predictive control literature (see [24]). The extra assumption that we use in this section is as follows:

Assumption 4.1 Suppose that $F$ is such that there exists a closed set $X_{f} \subset \mathbb{R}^{2 n}$ and a control law $u=u_{f}(\xi, x)$ such that the following conditions hold:

(i) $u_{f}(\xi, x) \in \mathbb{U}$, for all $(\xi, x) \in X_{f}$

(ii) if $(\xi, x) \in X_{f}$, then we have that $\left(G\left(\xi, u_{f}(\xi, x)\right), H(x)\right) \in X_{f}$;

(iii) for all $(\xi, x) \in X_{f}$ we have that

$$
F\left(G\left(\xi, u_{f}(\xi, x)\right), H(x)\right)-F(\xi, x) \leq-Q\left(\xi, x, u_{f}(\xi, x)\right) .
$$

Remark 4.2 We note that it is not necessary for $F$ to be a local control Lyapunov function in order to satisfy Assumption 4.1. Indeed, note that if $\ell(y, u) \geq \gamma(|y|)$ for some $\gamma \in \mathcal{K}$, then $\xi \neq x$ implies 

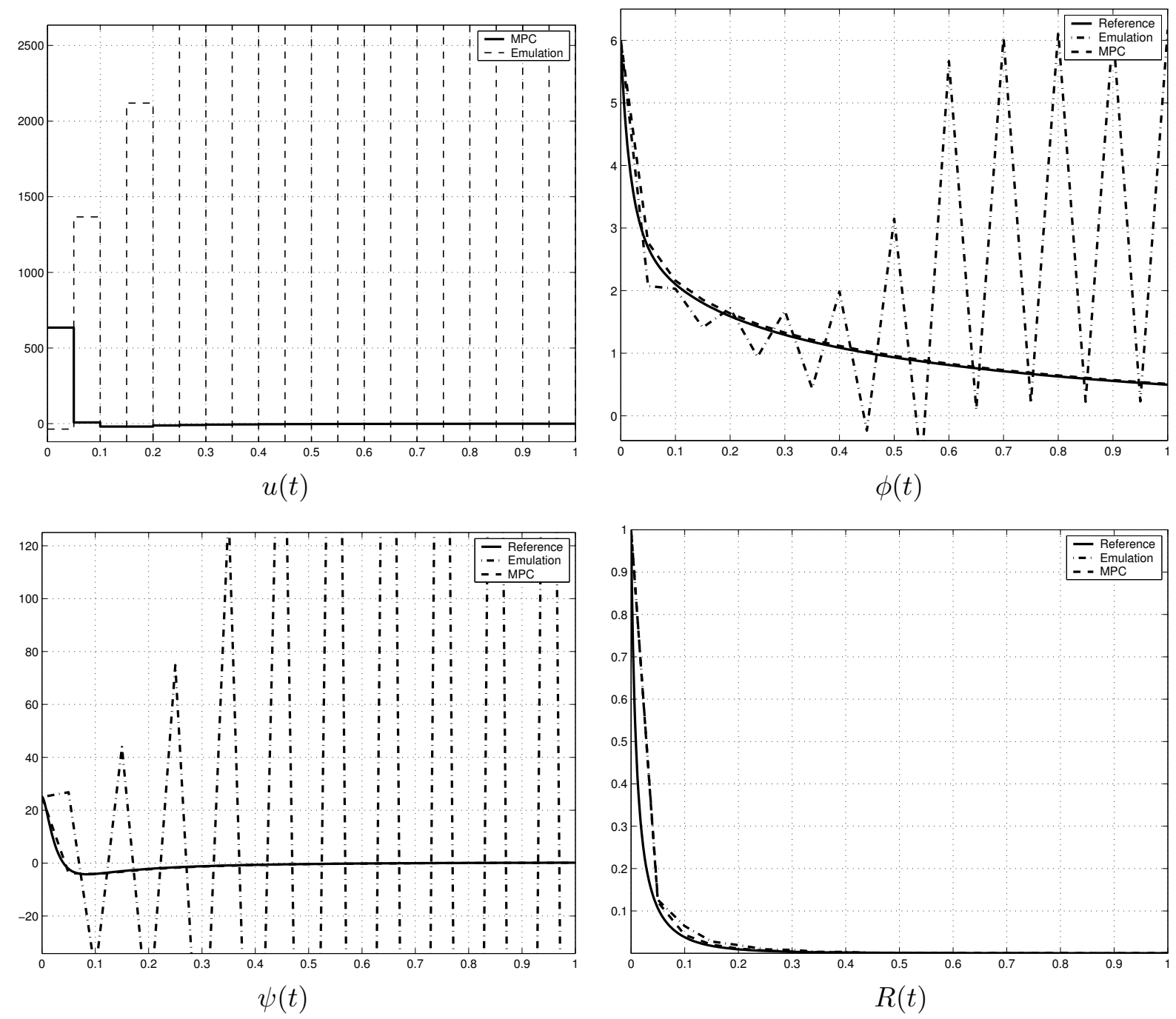

Figure 3.2: Improvement of MPC on unstable emulated controller

that $Q(\xi, x, u)>0$ (see the proof of Proposition 6.1 in the appendix). This, in turn implies

$$
\xi, x \in X_{f}, \xi \neq x \Longrightarrow F(\xi, x)>0 .
$$

Indeed, suppose Assumption 4.1 holds and that there exists $\xi^{*}, x^{*} \in X_{f}, \xi^{*} \neq x^{*}$ such that $F\left(\xi^{*}, x^{*}\right)=$ 0 . Then, we have that

$$
F\left(G\left(\xi^{*}, x^{*}\right), H\left(x^{*}\right)\right) \leq-Q\left(\xi^{*}, x^{*}, u_{f}\left(\xi^{*}, x^{*}\right)\right)<0,
$$

which is a contradiction since $F(\cdot, \cdot)$ is positive semi-definite. Hence, we can see that item (iii) of Assumption 4.1 implies that

(i) $F(0,0)=0$;

(ii) $\xi, x \in X_{f}$, and $\xi \neq x$ imply $F(\xi, x)>0$;

(iii) $\xi, x \in X_{f}$ and $\xi \neq x$ imply $F(G(\xi, x), H(x))-F(\xi, x)<0$.

Hence, in general $F$ is not a local control Lyapunov function for the system. However, it is straightforward to show that the function $U(x)+F(\xi, x)$ is a local control Lyapunov function for the system (2.13), (2.14) for any function $U(x)$ satisfying (3.2), (3.3). Moreover, we show next that if $F$ is a local control Lyapunov function for (2.13), (2.14) then under weak assumptions one can always find $\ell$ so that Assumption 4.1 holds. 
Theorem 4.3 Consider the discrete-time plant model (2.9). Suppose that Assumption 4.1 holds. Then, there exists a set $X_{M} \subset \mathbb{R}^{2 n}$ and a function $\mathcal{Q}: \mathbb{R}^{n} \times \mathbb{R}^{n} \times \mathbb{U} \rightarrow \mathbb{R}$, with $\mathcal{Q}\left(\xi, x, u_{M}\right) \geq$ $Q\left(\xi, x, u_{M}\right), \forall(\xi, x) \in X_{M}, u \in \mathbb{U}$ such that for all $(\xi, x) \in X_{M}$ we have that the controller (2.6) minimizes the following cost:

$$
\mathcal{J}\left(\xi, x, u_{[0, \infty)}\right):=\sum_{i=0}^{\infty} \mathcal{Q}\left(\xi_{i}, x_{i}, u_{i}\right)
$$

Proof of Theorem 4.3: From the principle of optimality we have that

$$
\begin{aligned}
V_{M}(\xi, x)= & Q\left(\xi, x, u_{M}(\xi, x)\right)+V_{M-1}\left(G\left(\xi, u_{M}(\xi, x)\right), H(x)\right) \\
= & V_{M}\left(G\left(\xi, u_{M}(\xi, x)\right), H(x)\right)+Q\left(\xi, x, u_{M}(\xi, x)\right) \\
& +\left[V_{M-1}\left(G\left(\xi, u_{M}(\xi, x)\right), H(x)\right)-V_{M}\left(G\left(\xi, u_{M}(\xi, x)\right), H(x)\right)\right] \\
= & V_{M}\left(G\left(\xi, u_{M}(\xi, x)\right), H(x)\right)+\mathcal{Q}\left(\xi, x, u_{M}(\xi, x)\right)
\end{aligned}
$$

where we denoted $\mathcal{Q}(\xi, x, u):=Q(\xi, x, u)+\left[V_{M-1}(G(\xi, u), H(x))-V_{M}(G(\xi, u), H(x))\right]$. Hence, we see that the controller $u_{M}(\xi, x)$ is indeed optimal for the newly defined cost. The last thing to prove is that

$$
V_{M-1}\left(G\left(\xi, u_{M}(\xi, x)\right), H(x)\right)-V_{M}\left(G\left(\xi, u_{M}(\xi, x)\right), H(x)\right) \geq 0 .
$$

Let the set $X_{M}$ be such that if $(\xi, x) \in X_{M}$, then $\left(\xi\left(t_{M}\right), x\left(t_{M}\right)\right) \in X_{f}$. Consider arbitrary $(\xi, x) \in X_{M}$. Suppose that the optimal sequence $\hat{u}_{[0, M-1]}=\hat{u}_{[0, M-1]}(\xi, x)$ that minimizes the cost $J_{M}\left(\xi, x, v_{[0, M-1]}\right)$ has been obtained. Let $\xi\left(t, \xi, \hat{u}_{[0, M-1]}\right)$ denote the optimal trajectory of the sampled data system. The controller $u=\hat{u}_{0}(\xi, x)$ steers the initial state $\xi$ to its successor state $\xi^{+}=\xi\left(T, \xi, \hat{u}_{0}(\xi, x)\right)=$ : $G\left(\xi, \hat{u}_{0}(\xi, x)\right)$. Denote also $x^{+}=x(T, x)=: H(x), x_{f}:=x\left(t_{M}, x\right)$ and $\xi_{f}:=x_{s}\left(t_{M}, \xi, \hat{u}_{[0, M-1]}\right)$. Since $\hat{u}_{[0, M-1]}=\left\{\hat{u}_{0}, \ldots, \hat{u}_{M-1}\right\}$ is an optimal sequence, $\left(\xi_{f}, x_{f}\right) \in X_{f}$ and also the sequence $\left\{\hat{u}_{1}, \ldots, \hat{u}_{M-1}\right\}$ steers $\xi^{+}$to $\xi_{f}$, we can add another element to this sequence to obtain another suboptimal control sequence $\tilde{u}:=\left\{\hat{u}_{1}, \ldots, \hat{u}_{M-1}, u_{f}(\xi, x)\right\}$ for the state $\left(\xi^{+}, x^{+}\right)$, where $u_{f}(\cdot, \cdot)$ comes from Assumption 4.1. Note that this control sequence because of Assumption 4.1 implies that $\left(G\left(\xi_{f}, u_{f}\left(\xi_{f}, x_{f}\right)\right), H\left(x_{f}\right)\right) \in$ $X_{f}$. Then, we can write:

$$
\begin{aligned}
J_{M}\left(\xi^{+}, x^{+}, \tilde{u}\right)= & V_{M-1}\left(G\left(\xi, \hat{u}_{0}\right), H(x)\right)+Q\left(\xi_{f}, x_{f}, u_{f}\left(\xi_{f}\right)\right) \\
& -F\left(\xi_{f}, x_{f}\right)+F\left(G\left(\xi_{f}, u_{f}\left(\xi_{f}\right)\right), H\left(x_{f}\right)\right) \\
\geq & V_{M}\left(G\left(\xi, \hat{u}_{0}\right), H(x)\right)
\end{aligned}
$$

for all $(\xi, x) \in X_{M}$, where the inequality follows from the item (iii) of Assumption 4.1. Since $u_{M}(\xi, x):=\hat{u}_{0}(\xi, x)$, this completes the proof.

The following proposition shows that Assumption 4.1 holds under relatively weak assumptions.

Proposition 4.4 Let $u_{f}(\xi, x)$ and $F(\xi, x)$ be such that items (i) and (ii) of Assumption 4.1 hold and, suppose that there exists $\alpha_{u} \in \mathcal{K}_{\infty}$ such that

$$
\left|u_{f}(\xi, x)\right| \leq \alpha_{u}(|(\xi, x)|) \quad \forall(\xi, x) \in X_{f} .
$$

Moreover, suppose that for all $(\xi, x) \in X_{f}$ we have

$$
\begin{aligned}
\alpha_{1}(|(\xi, x)|) & \leq F(\xi, x) \leq \alpha_{2}(|(\xi, x)|) \\
\Delta F:=F\left(G\left(\xi, u_{f}(\xi, x)\right), H(x)\right)-F(\xi, x) & \leq-\alpha_{3}(|(\xi, x)|)
\end{aligned}
$$

for some $\alpha_{1}, \alpha_{2}, \alpha_{3} \in \mathcal{K}_{\infty}$. Let $L>0$ be the Lipschitz constant for $(f(\xi, u), f(x, u(x)))$ on the set $X_{f} \times \mathbb{U}$. Then the item (iii) of Assumption 4.1 holds for any $\ell: \mathbb{R}^{2 n} \times \mathbb{U} \rightarrow \mathbb{R}_{\geq 0}$ such that:

$$
\ell(y, u) \leq \frac{1}{T} \alpha_{3} \circ \alpha\left(\frac{\exp (-L T)|y|}{2}+\frac{|u|}{2}\right) .
$$

where $\alpha(s):=1 / 2 \min \left\{s / 2, \alpha_{u}^{-1}(s / 2)\right\}$. 
Proof of Proposition 4.4: We use in this proof 1-norms, i.e. $|(\xi, x)|=|\xi|+|x|$. Let $L>0$ be the Lipschitz constant for $(f(\xi, u), f(x, u(x)))$ on the set $X_{f} \times \mathbb{U}$. Then, from invariance of $X_{f}$ we can write for all $(\xi, x) \in X_{f}$ that

$$
\begin{aligned}
|\xi(t)|+|x(t)| & \leq \exp (L T)(|\xi|+|x|)+(\exp (L T)-1)\left|u_{f}(\xi, x)\right| \\
& \leq \exp (L T)\left(|\xi|+|x|+\left|u_{f}(\xi, x)\right|\right) \quad \forall t \in[0, T] .
\end{aligned}
$$

Then we get directly from (4.6) and (4.4) and using the triangle inequality for $\mathcal{K}$ functions:

$$
\begin{aligned}
\Delta F & \leq-\alpha_{3}(|\xi|+|x|) \\
& \leq-\alpha_{3}\left(\frac{|\xi|+|x|}{2}+\frac{1}{2} \alpha_{u}^{-1}\left(\left|u_{f}(\xi, x)\right|\right)\right) \\
& \leq-\alpha_{3} \circ \alpha\left(|\xi|+|x|+\left|u_{f}(\xi, x)\right|\right) .
\end{aligned}
$$

Finally, we can use (4.8) to write

$$
\begin{aligned}
\Delta F & \leq-\frac{1}{T} \int_{0}^{T} \alpha_{3} \circ \alpha\left(|\xi|+|x|+\left|u_{f}(\xi, x)\right|\right) d t \\
& \leq-\frac{1}{T} \int_{0}^{T} \alpha_{3} \circ \alpha\left(\exp (-L T) \frac{|\xi(t)|+|x(t)|}{2}+\frac{\left|u_{f}(\xi, x)\right|}{2}\right) d t \\
& \leq-\frac{1}{T} \int_{0}^{T} \alpha_{3} \circ \alpha\left(\exp (-L T) \frac{|\xi(t)-x(t)|}{2}+\frac{\left|u_{f}(\xi, x)\right|}{2}\right) d t \\
& \leq-\int_{0}^{T} \ell\left(\xi(t)-x(t), u_{f}(\xi, x)\right) d t \\
& =-Q\left(\xi, x, u_{f}(\xi, x)\right)
\end{aligned}
$$

which completes the proof.

A simpler way to find appropriate $F$ and $\ell$ satisfying Assumption 4.1 is given below.

Corollary 4.5 Let $T>0$ be such that the $\xi$ subsystem in (2.9) is stabilizable. Then, given any $u_{f}(\xi)$ such that

$$
\xi^{+}=G\left(\xi, u_{f}(\xi)\right)
$$

is asymptotically stable and (4.4) holds, there exists $F$ so that Assumption 4.1 holds for any $\ell$ satisfying (4.7).

Sketch of proof of Corollary 4.5: Since (4.9) is stable, via converse Lyapunov theorems we can find a Lyapunov function $V_{1}(\xi)$ for this system. Moreover, since (2.14) is by assumption asymptotically stable, there exists a Lyapunov function $V_{2}(x)$ for this subsystem. Then, it is obvious that

$$
F(\xi, x):=V_{1}(\xi)+V_{2}(x)
$$

is a Lyapunov function for the overall system and the conclusion follows from Proposition 4.4.

\section{Conclusions}

We have considered implementation of continuous time control laws using a sampled feedback with a zero order hold assumption. The implementation aims at minimizing the distance between the trajectories of the sampled-data system and the continuous-time system in a receding horizon fashion. We have considered unconstrained model predictive control and under different conditions proved their stability, inverse optimality and feasibility properties. 


\section{References}

[1] B.D.O. Anderson, "Controller design: moving from theory to practice", IEEE Control Systems Magazine, vol. 13, no. 4, pp. 16-25, April, 1993.

[2] D. Angeli, E.D. Sontag and Y. Wang, "A characterization of integral input-to-state stability", IEEE Trans. Automat. Contr., vol. 45, pp. 1082-1097, 2000.

[3] A. Bemporad, A. Casavola and E. Mosca, "Nonlinear control of constrained linear systems via predictive reference management", IEEE Trans. Automat. Contr., vol. 42, pp. 340-349, 1996.

[4] F.H. Clarke, Y.S. Ledyaev, E.D. Sontag and A.I. Subbotin, "Asymptotic controllability implies feedback stabilization", IEEE Trans. Automat. Contr., vol. 42, pp. 1394-1407, 1997.

[5] E.G. Gilbert and I. Kolmanovsky, "Fast reference governors for systems with state and cobtrol constraints and disturbance inputs", Int. J. Robust Nonlin. Contr., vol. 9, pp. 1117-1141, 1999.

[6] G. Grimm, M.J. Messina, A.R. Teel and S. Tuna, "Model predictive control: for want of a local control Lyapunov function, all is not lost", to appear in IEEE Trans. Automat. Contr., 2004.

[7] L. Grüne, Asymptotic controllability and exponential stabilization of nonlinear control systems at singular points, SIAM J. Contr. Optimiz., 36 (1998), pp. 1485-1503.

[8] L. Grüne, Asymptotic behavior of dynamic and control systems under perturbation and discretization, Springer Verlag, Berlin, 2002.

[9] A. Isidori, Nonlinear control systems, 3rd Ed.. Springer-Verlag: London, 2002.

[10] A. Jadbabaie, J. Yu and J. Hauser, "Unconstrained receding horizon control of nonlinear systems", IEEE Trans. Automat. Contr., vol. 46, pp. 776-783, 2001.

[11] F. Camilli, L. Grüne and F. Wirth, "Control Lyapunov functions and Zubov's method", submitted, Trans. Amer. Math. Soc., 2004.

[12] T. Chen and B.A. Francis, Optimal sampled-data control systems. Springer-Verlag: London, 1995.

[13] H. Chen and F. Allgöwer, "A quasi-infinite horizon nonlinear model predictive control scheme with guaranteed stability", Automatica, vol. 34, pp. 1205-1217, 1998.

[14] G.F. Franklin, J.D. Powell and M. Workman, Digital control of dynamic systems, 3rd Ed. AddisonWesley, 1997.

[15] L. Grüne, D. Nešić and J. Pannek, "Model predictive sampled-data redesign for nonlinear systems", submitted to joint Conf. Decis. Contr. and Europ. Contr. Conf., Seville, Spain, 2005.

[16] D.S. Laila, D. Nešić and A.R. Teel, "Open and closed loop dissipation inequalities under sampling and controller emulation", Europ. J. Contr., vol. 8, No. 2, pp. 109-125, 2002.

[17] P. Katz, Digital control using microprocessors. Prentice Hall, 1981.

[18] C.M. Kellett, Advances in converse and control Lyapunov functions, PhD Thesis, University of California, Santa Barbara, June 2002.

[19] H.K. Khalil, Nonlinear systems, 3rd Ed.. Prentice Hall: New Jersey, 2002.

[20] M. Krstić, I. Kanellakopoulos, P. Kokotović, Nonlinear and Adaptive Control Design, John Wiley \& Sons Inc, 1995.

[21] R. Sepulchre, M. Janković and P.V. Kokotović, Constructive nonlinear control. Springer-Verlag: London, 1997. 
[22] A. Arapostathis, B. Jakubczyk, H.-G. Lee, S. Marcus and E.D. Sontag, "The effect of sampling on linear equivalence and feedback linearization", Syst. Contr. Lett., vol. 13, No. 5, December, pp. 373-381, 1989.

[23] L. Magni, G. Di Nicolao and R. Scattolini, "Output feedback and tracking of nonlinear systems with model predictive control", Automatica, vol. 37, pp. 1601-1607, 2001.

[24] D.Q. Mayne, J.B. Rawlings, C.V. Rao and P.O.M. Scokaert, "Contrained model predictive control: stability and optimality", Automatica, vol. 36, pp. 789-814, 2000.

[25] D. Nešić and A.R. Teel, "A framework for stabilization of nonliear sampled-data systems based on their approximate discrete-time models", to appear in IEEE Trans. Automat. Contr., 2002.

[26] D. Nešić, A.R. Teel and E.D. Sontag, Formulas relating $\mathcal{K} \mathcal{L}$ stability estimates of discrete-time and sampled-data nonlinear systems, Syst. Contr. Lett., vol. 38 (1999), pp. 49-60.

[27] D. Nešić, A.R. Teel and P.V. Kokotović, Sufficient conditions for stabilization of sampled-data nonlinear systems via discrete-time approximations, Sys. Contr. Lett., 38 (1999), pp. 259-270.

\section{Appendix}

Proposition 6.1 Assume that items (iii-a), (iii-b) and (iii-c) of Theorem 3.1 hold. Then, there exists $\alpha_{Q} \in \mathcal{K}_{\infty}$ such that:

$$
|x|+Q(\xi, x, u) \geq \alpha_{Q}(|(\xi, x)|) \quad \forall(\xi, x) \in \mathbb{R}^{2 n}, u \in \mathbb{U}
$$

To prove the above proposition, we first prove the following:

Fact: Assume that items (iii-a), (iii-b) and (iii-c) of Theorem 3.1 hold. Then for any sequence of initial values $\left(\xi_{j}, x_{j}\right), j \in \mathbb{N}$, with $\left|x_{j}\right|$ bounded and $\left|\xi_{j}\right| \rightarrow \infty$ we have

$$
Q\left(\xi_{j}, x_{j}, u\right) \rightarrow \infty \quad \forall u \in \mathbb{U} .
$$

Moreover, the convergence is independent of $u \in \mathbb{U}$.

Proof of the Fact: Consider a sequence $\left(\xi_{j}, x_{j}\right)$ meeting the assumptions. Since the continuous time closed loop system is asymptotically stable we find a constant $R>0$ such that $\left|x\left(t, x_{j}\right)\right| \leq R$ holds for all $j \in \mathbb{N}, t \geq 0$; without loss of generality we can assume $R \geq r_{0}$ for $r_{0}$ from the item (iii-b).

Now consider $j>0$ large enough such that $\left|\xi_{j}\right| \geq 9 R$ holds, which implies $\left|\xi_{j}-x_{j}\right| \geq 8 R$. Let $u \in \mathbb{U}$ be arbitrary and consider the solutions $\xi\left(t, \xi_{j}, u\right)$ of the sampled data system on the interval $[0, T]$. Now we consider two different cases:

Case 1: $\left|x\left(t, x_{j}\right)-\xi\left(t, \xi_{j}, u\right)\right| \geq\left|x_{j}-\xi_{j}\right| / 2$ for all $t \in[0, T]$.

In this case from the inequality $\ell(y, u) \geq \gamma(|y|)$ we immediately obtain

$$
Q\left(\xi_{j}, x_{j}, u\right) \geq T \gamma\left(\left|x_{j}-\xi_{j}\right| / 2\right) .
$$

Case 2: $\left|x\left(t, x_{j}\right)-\xi\left(t, \xi_{j}, u\right)\right|<\left|x_{j}-\xi_{j}\right| / 2$ for some $t \in[0, T]$. In this case let $\tau \in[0, T]$ be the minimal time for which this inequality holds. Then, since $\mid x_{j}-$ $x\left(t, x_{j}\right) \mid \leq 2 R$ and $2 R \leq\left|x_{j}-\xi_{j}\right| / 4$, this yields

$$
\begin{aligned}
\left|\xi\left(\tau, \xi_{j}, u\right)-\xi_{j}\right| & \geq\left|\xi_{j}-x\left(\tau, x_{j}\right)\right|-\left|x\left(\tau, x_{j}\right)-\xi\left(\tau, \xi_{j}, u\right)\right| \\
& \geq\left|\xi_{j}-x\left(\tau, x_{j}\right)\right|-\left|x_{j}-\xi_{j}\right| / 2 \\
& \geq\left|\xi_{j}-x_{j}\right|-2 R-\left|x_{j}-\xi_{j}\right| / 2 \\
& \geq\left|\xi_{j}-x_{j}\right|-\left|x_{j}-\xi_{j}\right| / 4-\left|x_{j}-\xi_{j}\right| / 2=\left|x_{j}-\xi_{j}\right| / 4
\end{aligned}
$$

This implies

$$
\int_{0}^{\tau}\left|f\left(\xi\left(t, \xi_{j}, u\right), u\right)\right| d t \geq\left|x_{j}-\xi_{j}\right| / 4
$$


Now the minimality of $\tau$ implies that for $t \in[0, \tau]$ we have

$$
\left|\xi\left(t, \xi_{j}, u\right)-x\left(t, x_{j}\right)\right| \geq\left|x_{j}-\xi_{j}\right| / 2 \geq R
$$

hence

$$
\left|\xi\left(t, \xi_{j}, u\right)\right| \leq\left|\xi\left(t, \xi_{j}, u\right)-x\left(t, x_{j}\right)\right|+R \leq 2\left|\xi\left(t, \xi_{j}, u\right)-x\left(t, x_{j}\right)\right|
$$

and thus the first inequality in the item (iii-b) implies

$$
\ell\left(\left|\xi\left(t, \xi_{j}, u\right)-x\left(t, x_{j}\right)\right|, u\right) \geq\left|f\left(\xi\left(t, \xi_{j}, u\right), u\right)\right| .
$$

This yields

$$
\begin{aligned}
Q\left(\xi_{j}, x_{j}, u\right) & \geq \int_{0}^{\tau} \ell\left(x\left(t, x_{j}\right)-\xi\left(t, \xi_{j}, u\right), u_{0}\right) d t \\
& \geq \int_{0}^{\tau}\left|f\left(\xi\left(t, \xi_{j}, u\right), u\right)\right| d t \\
& \geq\left|x_{j}-\xi_{j}\right| / 4
\end{aligned}
$$

Now, in both cases we have found a lower bound for $Q\left(\xi_{j}, x_{j}, u\right)$ which is independent of $u$ and tends to $\infty$ as $j \rightarrow \infty$. This shows the fact.

Proof of Proposition 6.1: First note that $Q(\xi, x, u)>0$ whenever $\xi \neq x$. Indeed, whenever $\xi \neq x$, there exists $\tau=\tau(\xi, x)>0$ such that $|\xi(s, \xi, u)-x(s, x)|>0, \forall s \in[0, \tau]$ and hence we can write:

$$
Q(\xi, x, u)=\int_{0}^{T} \ell(\xi(s, \xi, u)-x(s, x), u) d s \geq \int_{0}^{\tau} \gamma(|\xi(s, \xi, u)-x(s, x)|) d s>0 .
$$

Moreover, it is obvious that $Q(0,0, u) \geq 0, \forall u \in \mathbb{U}$. Note that this implies that $|x|+Q(\xi, x, u)$ can be lower bounded by a positive definite function of $(\xi, x)$. Moreover, consider a sequence of initial values $\left(\xi_{j}, x_{j}\right), j \in \mathbb{N}$, with $\left|\left(\xi_{j}, x_{j}\right)\right| \rightarrow \infty$ as $j \rightarrow \infty$. Then if $\left|x_{j}\right| \rightarrow \infty$ we have

$$
\left|x_{j}\right|+Q\left(\xi_{j}, x_{j}, u\right) \rightarrow \infty .
$$

On the other hand, if $x_{j}$ is bounded then we have that $\left|\xi_{j}\right| \rightarrow \infty$ and using the above Fact we have $Q\left(\xi_{j}, x_{j}, u\right) \rightarrow \infty$ which again implies that (6.1) holds. Since $|x|+Q(\xi, x, u)$ is a continuous function of its arguments and it is radially unbounded in $(\xi, x)$, it follows from [19, Lemma 4.3] that there exists $\alpha_{Q} \in \mathcal{K}_{\infty}$ such that

$$
|x|+Q(\xi, x, u) \geq \alpha_{Q}(|(\xi, x)|) \quad \forall(\xi, x) \in \mathbb{R}^{2 n}, u \in \mathbb{U},
$$

which completes the proof.

Proposition 6.2 Suppose that the items (iii-a), (iii-b) and (iii-c) of Theorem 3.2 hold. Then, there exists $D>0$ and $a>0$ such that:

$$
|x|^{2}+Q(\xi, x, u) \geq a|(\xi, x)|^{2} \quad \forall|(\xi, x)| \leq D, u \in \mathbb{U} .
$$

Moreover, if all the assumptions hold globally (i.e. the items (iii-a), (iii-b) and (iii-c) of Theorem 3.3 hold), then (6.2) holds for all $(\xi, x) \in \mathbb{R}^{2 n}$ and $u \in \mathbb{U}$.

Proof of Proposition 6.2: In this proof we use the 2-norm (Euclidean norm) for all vectors. Let $D>0$ and $t_{1}>0$ be such that for all $|(\xi, x)| \leq D$ and $u \in \mathbb{U}$ we have that $\max \{|\xi(t)|,|x(t)|\} \leq \frac{\Delta_{\ell}}{2}$ for all $t \in\left[0, t_{1}\right]$. Note that this implies that $|\xi(t)-x(t)| \leq \Delta_{\ell}, \forall t \in\left[0, t_{1}\right]$. Since $f$ and $u$ are locally Lipschitz, it is always possible to find such $D$ and $t_{1}$. Let $L>0$ be such that

$$
|f(x, u)| \leq L(|x|+|u|) \quad \forall|x| \leq \Delta_{\ell}, u \in \mathbb{U}
$$

and

$$
|f(x, u(x))| \leq L|x| \quad \forall|x| \leq \Delta_{\ell} .
$$


It is a standard exercise to show that

$$
|x(t, x)| \leq e^{L t}|x|, \quad \forall t \in\left[0, t_{1}\right], x \in B_{D} .
$$

Moreover, we also have for all $(\xi, x) \in B_{D}, u \in \mathbb{U}$ and $t \in\left[0, t_{1}\right]$ that:

$$
\begin{aligned}
\left|\frac{d}{d t} \xi^{T}(t) \xi(t)\right| & =\left|2 \xi^{T}(t) f(\xi(t), u)\right| \\
& \leq 2 L\left(|\xi(t)|^{2}+|\xi(t)| \cdot|u|\right) \\
& \leq 2 L\left(3|\xi(t)|^{2}+2|u|^{2}\right) \\
& \leq 6 L\left(|\xi(t)|^{2}+|u|^{2}\right)
\end{aligned}
$$

which implies that

$$
\frac{d}{d t} \xi^{T}(t) \xi(t) \geq-6 L\left(|\xi(t)|^{2}+|u|^{2}\right)
$$

Using standard comparison theorems and the fact that $|\xi(t)| \geq 0, \forall t$, one can show that this implies that

$$
|\xi(t)|^{2} \geq \max \left\{\exp (-6 L t)|\xi|^{2}+(\exp (-6 L t)-1)|u|^{2}, 0\right\}=: b(t, \xi, u) .
$$

Suppose that $t_{2}$ is such that

$$
a_{\ell} \cdot t_{2} \cdot \exp \left(2 L \cdot t_{2}\right) \leq \frac{1}{2}
$$

Note that

$$
(\max \{a-b, 0\})^{2}+b^{2} \geq \frac{a^{2}}{4} \quad \forall a, b \geq 0 .
$$

Using (6.5) and the definition of $b(t, \xi, u)$ we can write:

$$
\begin{aligned}
b\left(t_{2}, \xi, u\right)+|u|^{2} & =\max \left\{\exp \left(-6 L t_{2}\right)|\xi|^{2}+\left(\exp \left(-6 L t_{2}\right)-1\right)|u|^{2}, 0\right\}+|u|^{2} \\
& \geq \exp \left(-6 L t_{2}\right)|\xi|^{2}+\exp \left(-6 L t_{2}\right)|u|^{2} \\
& \geq \exp \left(-6 L t_{2}\right)|\xi|^{2}
\end{aligned}
$$

Finally, using (6.4) and (6.5), we can write:

$$
\begin{aligned}
|x|^{2}+Q(\xi, x, u) & =|x|^{2}+\int_{0}^{T} \ell(\xi(t)-x(t), u) d t \\
& \geq|x|^{2}+a_{\ell} \int_{0}^{T}\left(|\xi(t)-x(t)|^{2}+|u|^{2}\right) d t \\
& \geq|x|^{2}+a_{\ell} \int_{0}^{t_{2}}\left((\max \{|\xi(t)|-|x(t)|, 0\})^{2}+u^{2}\right) d t \\
& \geq|x|^{2}+a_{\ell} \cdot t_{2}\left(\left(\max \left\{\sqrt{b\left(t_{2}, \xi, u\right)}-\exp \left(L t_{2}\right)|x|, 0\right\}\right)^{2}+|u|^{2}\right) \\
& \geq \frac{1}{2}|x|^{2}+a_{\ell} \cdot t_{2} \cdot\left(\exp \left(2 L t_{2}\right)|x|^{2}+\left(\max \left\{\sqrt{b\left(t_{2}, \xi, u\right)}-\exp \left(L t_{2}\right)|x|, 0\right\}\right)^{2}+|u|^{2}\right) \\
& \geq \frac{1}{2}|x|^{2}+a_{\ell} \cdot t_{2} \cdot\left(\frac{b\left(t_{2}, \xi, u\right)}{4}+|u|^{2}\right) \\
& \geq \frac{1}{2}|x|^{2}+\frac{a_{\ell} \cdot t_{2} \cdot \exp \left(-6 L t_{2}\right)}{4}|\xi|^{2} \\
& \geq a|(\xi, x)|^{2},
\end{aligned}
$$

where

$$
a:=\min \left\{\frac{1}{2}, \frac{a_{\ell} \cdot t_{2} \cdot \exp \left(-6 L t_{2}\right)}{4}\right\} .
$$

Obviously, if all conditions hold globally then the result is global. 